A. Pramesh Rao, G. Swarup and Gopal-Krishna, eds.

\title{
Probes of Low Surface Brightness Galaxies through Low Frequency Spectroscopy with GMRT
}

\author{
D. Narasimha and S.M. Chitre \\ Tata Institute of Fundamental Research, Homi Bhabha Road, \\ Mumbai 400005, India
}

\begin{abstract}
Low Surface Brightness galaxies (LSBs) may well constitute an important component of the Universe and a good fraction of baryons may even be locked up in their gravitational potential. Existence of such massive objects was predicted from an investigation of gravitational lens systems more than a decade ago (Narasimha \& Chitre, 1989). There are more compelling reasons for believing their universality based on the of the analysis of the images around the cusp caustics in some of the wellstudied lenses. A complete sample of the mass, size and rotation velocity of LSBs in our neighbourhood, as well as in a limited volume at a larger redshift will be important for estimation of power spectrum as well as evolution of structures in the Universe. The GMRT type instrument is ideally suited for detection and study of the LSBs. We expect to detect $21 \mathrm{~cm}$ emission from around 2 to $10 \mathrm{LSBs}$ per beam of GMRT dish at the level of $5 \mathrm{mJy}$ flux with a survey covering $30 \mathrm{Mhz}$ bandwidth, if their line width is of the order of $30 \mathrm{~km} / \mathrm{sec}$.
\end{abstract}

\section{Introduction}

The mass density of matter in the Universe is believed to be at least $20 \%$ of the closure density, out of which the baryonic matter appears to constitute no more than $25 \%$. At the scale of galaxy-clusters, $\Omega \sim 0.1$, but only approximately $10 \%$ of it is in the form of gas which manifests through its X-ray emission. There is considerably less mass in the luminous member galaxies and the bulk of the contribution to its gravitational potential is from unseen matter. On galaxy-scales, the discrepency becomes worse with galactic luminous matter constituting less than $1 \%$ of the closure density. This is far less than the baryonic density inferred from primordial nucleosynthesis. Consequently, it is evident that there should be substantial amount of matter, mostly baryonic, at galactic to cluster scales. Efforts like MACHO projects to detect them in compact objects in the halo of galaxies have not been very fruitful. Methods should be developed, not primarily dependent upon optical flux from the source, but that could still be a reliable probe to directly sample this unaccounted for mass.

In the last decade Malin developed image enhancing techniques using which he could identify features in the images hitherto unnoticed (cf. Malin, 1993). Consequently, a survey to detect nearby Low Surface Brightness Galaxies (LSBs) was undertaken. Ever since the detection of Malin 1 and similar massive LSBs 
(Bothun et al, 1987), many research groups have been carrying out detailed observational programmes to detect LSBs, which appear to be normal galaxies but with a considerably reduced rate of star formation. The amount of baryonic matter in these galaxies is still uncertain. Zwaan et al(1997) carried out a strip survey with Arecibo and follow-up observations with VLA to prepare a catalogue of LSBs. They argued that the baryonic content of these galaxies should be $\Omega_{H I} \sim 4 \times 10^{-4}$. Sprayberry et al(1996) argue that without incompleteness correction due to the faintness of these objects, their number density could be substantially underestimated, though they do not believe that HI in LSBs could make for for a major fraction of baryons in the Universe. They, in fact suggest that the number of LSBs could be comparable to that of normal disk galaxies. Their best fitting Schecter luminosity function

$$
\phi(M) d M=\phi^{*}\left(\frac{L}{L^{*}}\right)^{\alpha+1} \exp -\left(\frac{L}{L^{*}}\right) d M
$$

had the following parameters:

$$
\alpha=-1.4 \text { and } \phi^{*}=0.0036 h^{3} M p c^{-3} \text { for B magnitude, } M_{B}^{*}=-18.3 .
$$

Evidently, the estimate of power spectrum from this fit will involve an assumption about the mass to light ratio for the LSBs.

It has been suggested that the faint blue objects detected by HST could be the counterparts of LSBs. Ferguson \& McGaugh (1995) argued that the color and luminosity function of the faint blue sample can be explained without invoking any drastic evolution in normal galaxies, if we assume the nearby LSBs are their unevolving local counterparts. However, we would almost certainly need special observing plans to detect LSBs that could correspond to objects with surface brightness fainter than $24 \mathrm{~B}$ mag $\operatorname{arcsec}^{-2}$.

\section{Results from Gravitational Lens Analysis}

Gravitational lens offers an independent method to trace mass distribution in the Universe. The systems detected from complete samples obtained from $\mathrm{ra}$ dio survey can be especially considered to be good tracers of gravitating mass confined in bound objects over $10^{10}$ to $10^{13} M_{\odot}$ scale. Last decade, Narasimha \& Chitre constructed models for most of the then known gravitational lens systems using VLBI magnification matrices and examined whether the lens galaxy should have been detected. They argued that at least three among a dozen systems then known are probably lensed by dark objects. From an estimate of the mass, scale-length and favoured density profiles, they suggested that the lens could have concentrated central density structure due to which star formation in the disk might not have been favoured. On this basis, they predicted that such lenses could be detectable through their hydrogen emission, specifically the $21 \mathrm{~cm}$ radio emission (Narasimha \& Chitre, 1989). Since then an X-ray cluster of temperature around $8.6 \mathrm{kev}$ was detected (Hattori et al, 1997 ) with scale lengths similar to the predicted value (Narasimha et al, 1987). But optically, only a massive elliptical galaxy could be found at the expected 
position. In another system, $2345+007$, though there is possible evidence for distortion of background sources due to weak lensing, no lens galaxy or cluster could be located. Narasimha \& Chitre also examined the models as well as absorption line strengths in many lens systems to surmise that some of the absorption lines may be due to unobserved lens galaxies. There are reasons to invoke dark extended mass distribution around lens galaxies even in well-studied systems like $1422+231,1115+080$.

Based on our experience with modeling the gravitational lens systems, we speculate that LSBs and normal young galaxy-clusters have similar structure. An important result from our lens programme is that the core radius of the gravitational mass of lenses producing images over a range of scales between 2 arcseconds to a fraction of an arcminute, have practically similar value, of the order of 10 to $30 \mathrm{kpc}$, though the observed scale length of the luminous matter spans a considerably wider range of less than $2 \mathrm{kpc}$ to more than a megaparsec. These were the main features of the LSB models predicted by Narasimha \& Chitre, purely based on gravitational lensing:

a. A central mass condensation which is likely to have values of velocity dispersion larger than normal galaxies, but smaller scale lengths.

b. An extended mass distribution over considerably larger scale length.

c. Hydrogen emission from the object as tracer of the baryonic matter in the mass condensate.

Consequently, these are the features we expect in an LSB:

a. A cusp-like bulge,

b. a baryonic gas component which will have a shallower density profile as the object evolves,

c. the optically luminous matter, which depends on the star formation history of the core.

Malin 1, for example, has a bulge with central surface brightness of around $20 \mathrm{mag} \operatorname{arcsec}^{-2}$, falling off to nearly $23 \mathrm{mag} \operatorname{arcsec}^{-2}$ at few kiloparsecs. But the disk has a scale length of $55 \mathrm{kpc}$, central surface brightness of 25.7 mag $\operatorname{arcsec}^{-2}$ and HI mass of around $10^{11} M_{\odot}$. The $21 \mathrm{~cm}$ hydrogen emission is 3.5 $\mathrm{Jy} \mathrm{km} \mathrm{sec}-1$, but the $20 \%$ linewidth of the emission is $341 \mathrm{~km} \mathrm{sec}^{-1}$.

\section{Expectations from a GMRT-type Instrument}

GMRT has the following advantages for carrying out a survey of LSBs:

Special $21 \mathrm{~cm}$ feeder having frequency coverage below the Galactic $21 \mathrm{~cm}$ down to redshift of nearly 0.4 .

Frequency resolution closely matching the expected line width from typical LSBs and bandwidth wide enough to carry the survey with minimal exposure to the same region. 
Utility of single outer dish when the inner ones in the square array alone are used for interferometry.

A single GMRT dish has half opening angle of $21^{\prime}$ for the $21 \mathrm{~cm}$ feeder. The volume covered within a distance of $100 \mathrm{Mpc}$ within one beam is approximately $125 \mathrm{Mpc}^{3}$. The minimum mass of neutral hydrogen in this volume, confined in LSB type objects, based on the existing surveys should be $2 \times 10^{9} M_{\odot}$. The number of LSBs, however, will strongly depend on the lower mass cut off in the Schecter function. But taking a slight optimistic view, we could hope that a good fraction of this mass may be residing in galaxies of mass $10^{9}$ solar mass, consistent with the catalogue of Zwaan et al.(1997). If the rotation velocity of the galaxy having this mass range were around $30 \mathrm{~km} / \mathrm{sec}$ and $\mathrm{HI}$ mass, about $2 \times 10^{8} M_{\odot}$, the expected $21 \mathrm{~cm}$ line emission will be $5 \mathrm{mJy}$.

Consequently, we could expect of the order of 2 to 10 LSBs with fluxes around 2 to $10 \mathrm{mJy}$, as reasonable, though slightly optimistic estimates. This is not inconsistent with the existing survey, and is a lower limit based on the estimates derived from gravitational lensing (if our assumptions on lensing by LSBs are correct). However, the major difficulty in the detection of LSBs will be the contamination due to emission from normal galaxies. This requires follow up observations of the local candidate regions with specially tuned wavelength bands.

Detection of a sample of optically faint galaxy population having a deep central potential and a shallow extended region of hydrogen emission will have far reaching implications for the theory of structure formation in the Universe.

Acknowledgments. We thank Govind Swarup and Jayaram Chengalur for discussions regarding ongoing surveys of LSBs and feasibility of using GMRT.

\section{References}

Bothun, G., et al. 1987, AJ, 94, 23

Ferguson, H.C. \& McGaugh, S.S. 1995, ApJ, 440, 470

Hattori, M. et al. 1997, Nature , 388, 146

Malin, D. 1993, in A view of the Universe, Sky Publ Corp

Narasimha, D., \& Chitre, S.M. 1989, AJ, 97, 327

Narasimha, D., Subramanian, K., \& Chitre, S.M. 1987, ApJ, 315, 434

Sprayberry, D. et al. 1996, in IAU Symp. 171, New light on Galaxy evolution, eds. R. Bender \& R.L. Davies, 446

Zwaan, M.A. et al. 1997, ApJ, 490, 173. 